\title{
Exclusive Charmless $B$ Decays in QCD
}

\section{SI-HEP-2006-08}

\author{
Alexander Khodjamirian ${ }^{\text {a* }}$ \\ a Theoretische Physik 1, Fachbereich Physik, \\ Universität Siegen, D-57068 Siegen, Germany
}

The problem of hadronic input in charmless nonleptonic $B$ decays is discussed. QCD sum rules and their light-cone versions (LCSR) provide an important part of this input, such as the decay constant $f_{B}$ and $B \rightarrow \pi$ form factor. Employing the LCSR technique, the $B \rightarrow \pi \pi$ hadronic matrix elements with emission, penguin and annihilation topologies are calculated, with no evidence for large nonfactorizable effects and/or strong phases.

\section{INTRODUCTION}

Charmless hadronic $B$ decays proceed due to a unique interference of electroweak and strong interactions, allowing one to observe CP-violation effects within and beyond Standard Model (discussed in the talks by M. Gronau and R. Fleischer). On the experimental side, data on branching fractions and CP-asymmetries are being steadily accumulated [1] in many $B \rightarrow h_{1} h_{2}$ channels $\left(h_{1,2}=\pi, K, \rho, K^{*}\right.$ and other light mesons). Still there are noticeable differences between BaBar and Belle results. On the theory side, there is a big challenge of calculating the relevant hadronic input in QCD, which is the main topic of my talk.

\section{THEORY INPUTS IN CHARMLESS $B$ DECAYS}

Integrating out the electroweak and quarkgluon interactions at short distances and constructing the effective weak Hamiltonian, one arrives at a generic expression for the charmless decay amplitude, e.g., for $\bar{B}^{0} \rightarrow \pi^{+} \pi^{-}$:

$$
\begin{array}{r}
A\left(\bar{B}^{0} \rightarrow \pi^{+} \pi^{-}\right)=\left\langle\pi^{+} \pi^{-}\left|H_{e f f}\right| \bar{B}^{0}\right\rangle \\
=\frac{G_{F}}{\sqrt{2}} \sum_{T=E, P_{c}, A, . .} \sum_{i} \lambda_{i} c_{i}(\mu)\left\langle\pi^{+} \pi^{-}\left|O_{i}\right| \bar{B}^{0}\right\rangle_{\mu}^{T},
\end{array}
$$

\footnotetext{
* Invited talk at the First Workshop on Theory, Phenomenology and Experiments in Heavy Flavour Physics, Anacapri, Italy, May 29-31 2006.
}

where $\lambda_{i}$ and $c_{i}$ are the CKM factors and Wilson coefficients, respectively, and $\mu \sim m_{b}$ is the renormalization scale for $H_{\text {eff }}$. Each operator $O_{i}$ in the effective Hamiltonian generates several hadronic matrix elements with different topologies $T$, that is, the ways of contracting the quark fields in the initial $B$ meson and final pions. Here, $T=$ $E, P_{c}, A, .$. , denotes emission, charming penguin, annihilation, etc. Similar to Eq.(1), the hadronic input for each nonleptonic channel $B \rightarrow h_{1} h_{2}$ is encoded in a set of process-dependent matrix elements $\left\langle h_{1} h_{2}\left|O_{i}\right| B\right\rangle^{T}$. Their phases interfere with the CKM phase contained in some of $\lambda_{i}$ 's.

One can in principle avoid a direct calculation of separate matrix elements and employ isospin and $S U(3)_{f l}$ symmetries. The combinations of the above hadronic matrix elements form a smaller set of independent flavour-symmetry amplitudes. The latter are then fitted from the measured experimental observables and used to predict other observables (see e.g., 2 and the talk by F. Schwab). Amplitude decompositions and fits, being useful phenomenological tools, provide very limited information on the details of the quark-gluon dynamics in hadronic $B$ decays. To clarify the role of emission, penguin and annihilation topologies and to assess the magnitude of $S U(3)_{f l}$-symmetry violation in charmless channels, one has to apply calculational methods based on QCD.

Amplitudes with two hadrons in the final state are not directly accessible in lattice QCD. To calculate the hadronic matrix elements for $B \rightarrow$ 
$h_{1} h_{2}$, one employs approximations and effective theories valid in $m_{b} \rightarrow \infty\left(E_{h 1, h 2} \sim m_{b} / 2 \rightarrow \infty\right)$ limits. The $1 / m_{b}\left(1 / E_{h}\right)$ expansion is combined with the perturbation theory in $\alpha_{s}$. The QCD factorization (QCDF) approach [3], the framework of SCET 4, or a combination of both (see e.g., [5]) are used. In these approaches the input includes heavy-to-light form factors (e.g., the $B \rightarrow \pi$ form factor at small momentum transfer $\left.f_{B \pi}^{+}(0)\right)$, as well as the light-cone distribution amplitudes (DA's) of $h_{1}, h_{2}$ and the inverse moment $\lambda_{B}$ of the B-meson DA. For example, in the original QCDF analysis of $B \rightarrow K \pi, \pi \pi$ decays [3] the following parameters were adopted:

$f_{B \pi}^{+}(0)=0.28 \pm 0.05, \lambda_{B}=350 \pm 150 \mathrm{MeV}$,

in agreement with the QCD sum rule predictions (see the next section). A different approach is PQCD [6] where the form factors are assumed to have a perturbative expansion and the meson wave functions depend on transverse momenta.

Successful phenomenological applications of QCDF or SCET to $B \rightarrow h_{1} h_{2}$ are possible if all $1 / m_{b}$ effects are under control. In fact, in QCDF the annihilation contribution to $B \rightarrow \pi \pi$, as well as the twist-3 part of the spectator-scattering (part of the emission topology) contain end-point singularities. In the "default version" of QCDF [3] these contributions are parameterized with complex amplitudes, in other words, by additional input parameters. The latter are expected to be reasonably small with respect to the leading factorizable amplitude. Meanwhile, the current $B \rightarrow \pi \pi$ data, in particular, the unexpectedly large $\mathrm{BR}\left(B^{0} \rightarrow \pi^{0} \pi^{0}\right)$ and direct CP-asymmetry in $B^{0} \rightarrow \pi^{+} \pi^{-}$(observed by Belle), cannot be reproduced without inflating at least one of the hadronic matrix elements (together with its phase) in the decomposition (2). The uncalculable $O\left(1 / m_{b}\right)$ complex amplitudes mentioned above offer one possibility which, however would mean a rather poor $1 / m_{b}$ expansion. Another possibility [5] is that the NLO, $O\left(\alpha_{s}^{2}\right)$ spectatorscattering effects (not suppressed at $m_{b} \rightarrow \infty$ ) are large. In this case, a better agreement with the $B \rightarrow \pi \pi$ data is achieved, if both the form factor and inverse moment in (2) become numerically close to their lower limits.
A different point of view is adopted in the recent phenomenological analyses based on SCET [4, where the charming penguin contribution is allowed to vary (resembling the ansatz suggested earlier in [7]). The fit to the $B \rightarrow \pi \pi$ data in the SCET approach yields a large strong phase for the penguin amplitude and a form factor smaller than in (2): $f_{B \pi}^{+}(0)=(0.19 \pm 0.01 \pm 0.03)(4.25 \times$ $10^{-3} /\left|V_{u b}\right|$ ). In addition, according to $[8$ (see also the talk by Z. Ligeti), the annihilation contribution in SCET is finite and real.

Summarizing this brief overview, so far there is no consensus on the hadronic input in charmless $B$ decays. The methods based on $1 / m_{b}$ expansion are not in a position to reproduce all $B \rightarrow \pi \pi$ amplitudes in agreement with the current data, a situation sometimes called the " $B \rightarrow \pi \pi$ puzzle". Only if some of the hadronic matrix elements (leading or suppressed in $1 / m_{b}$ ) are varied as free parameters, successful fits to $B \rightarrow \pi \pi$ and, eventually also to $B \rightarrow K \pi$ data are achieved. (A more detailed analysis can be found, e.g., in [9].)

In what follows, I will focus on a few important questions arising from the above discussion: Are the values of $f_{B \pi}(0)$ and $\lambda_{B}$ smaller than expected earlier? How large are the $S U(3)_{f l}$ violation effects in the form factors? Is it possible to calculate the hadronic matrix elements $\left\langle h_{1} h_{2}\left|O_{i}\right| B\right\rangle^{T}$ at finite $m_{b}$ and assess the role of various quark topologies (emission, penguin, annihilation)? I will address these questions using the results obtained from QCD sum rules and LCSR.

\section{USE OF QCD SUM RULES AND LCSR}

\subsection{The $B$ meson decay constant}

The decay constant $f_{B}$ has no direct relation to charmless $B$ decays, but plays an important role for the normalization of other hadronic matrix elements, The recent first measurement of $B R\left(B \rightarrow \tau \nu_{\tau}\right)$ determining $\left|V_{u b}\right| f_{B}$, makes the size of this constant an even more topical issue. The QCD sum rule for $f_{B}^{2}$ is obtained from the vacuum correlation function of two heavy-light currents calculated with the local (condensate) operator-product expansion (OPE) and, primar- 
ily, at a finite $b$-quark mass. Importantly, it is also possible to derive the sum rule in HQET for the "static" constant $\hat{f}_{B} \simeq f_{B} \sqrt{m_{b}}$. The updated sum rule determination of $f_{B}$ with $O\left(\alpha_{s}^{2}\right)$ accuracy can be found in the two independent analyses [10] and [11, predicting $f_{B}=210 \pm 19$ $\mathrm{MeV}, f_{B_{s}}=244 \pm 21 \mathrm{MeV}$ and $f_{B}=206 \pm 20$ $\mathrm{MeV}$, respectively. To compare, the recent lattice $\left(n_{f}=3\right)$ result is [12] $f_{B}=216 \pm 9 \pm 19 \pm 7$ $\mathrm{MeV}, f_{B_{s}}=260 \pm 7 \pm 26 \pm 9 \mathrm{MeV}$ (see also the talk by $\mathrm{V}$. Lubicz). The first measurement of $B R\left(B \rightarrow \tau \nu_{\tau}\right)$ reported by Belle Collaboration [13] yields $\left|V_{u b}\right| f_{B}=\left(7.73_{-1.02-0.58}^{+1.24+0.66}\right) \times 10^{-4} \mathrm{GeV}$. Using the value $\left|V_{u b}\right|=(4.39 \pm 0.19 \pm 0.27) \times 10^{-3}$ [1] based on the inclusive $b \rightarrow u$ analysis, one obtains $f_{B}=176_{-23-19}^{+28+20} \mathrm{MeV}$. There is an agreement with the above QCD predictions within the experimental errors and theoretical uncertainties. Note, however, that if one uses the central value of the experimental result and the calculated $f_{B}$, a smaller central value for $\left|V_{u b}\right|$ is obtained.

\subsection{Heavy-to-light Form Factors}

The LCSR for the $B \rightarrow \pi$ form factor $f_{B \pi}^{+}\left(q^{2}\right)$ is derived from the vacuum-pion correlation function expanded near the light-cone in terms of the pion DA's. The latter are determined from the two-point QCD sum rules and/or from the LCSR for the pion e.m. form factors. For consistency, the value of $f_{B}$ and the quark-hadron duality threshold $s_{0}^{B}$ are taken from the sum rule discussed in the previous subsection. The recent LCSR result [14]

$f_{B \pi}^{+}(0)=0.258 \pm 0.031$

was obtained with the NLO, $O\left(\alpha_{s}\right)$ (in the twist 2,3 parts) and LO (in the suppressed twist-4 part) accuracy. From this result and the HFAG average of $B R\left(B \rightarrow \pi l \nu_{l}\right)$ at $0<q^{2}<16 \mathrm{GeV}^{2}$ the value $\left|V_{u b}\right|=3.25 \pm(0.17)_{\exp }\left({ }_{-0.36}^{+0.54}\right)_{t h} \times 10^{-3}$ is extracted [1. It is tempting to say that this value is smaller than the "inclusive" one, and closer to $\left|V_{u b}\right|$ obtained from the central value of $B R\left(B \rightarrow \tau \nu_{\tau}\right)$ combined with the QCD prediction for $f_{B}$. However, the errors and uncertainties are still too large for a definitive comparison. A value of the $B \rightarrow \pi$ form factor

$f_{B \pi}^{+}(0)=0.26 \pm 0.02 \pm 0.03$ close to (3) was reproduced from the same LCSR in 15 (without the small twist-3 $O\left(\alpha_{s}\right)$ correction). Here the uncertainties induced by the pion DA's and by other sum rule parameters are shown, respectively.

The $B \rightarrow \pi$ form factor is accessible on the lattice only at sufficiently large $q^{2}$. It is possible to use dispersion bounds to extrapolate the lattice QCD results to $q^{2}=0$. The two recent analyses yield $f_{B \pi}^{+}(0)=0.25 \pm 0.06$ (the version without the SCET point) [16] and $f_{B \pi}^{+}(0)=0.25 \pm 0.04$ [17, in a good agreement with the above LCSR results. The interval of the form factor advocated by the SCET fits to $B \rightarrow \pi \pi$ still agrees with both LCSR and lattice QCD, but only within uncertainties.

Combining the average $B R\left(B \rightarrow \pi l \nu_{l}\right)=$ $(1.34 \pm 0.08 \pm 0.08) \times 10^{-4}[1$, the slope parameter $\alpha_{B \pi}=0.61 \pm 0.09$ fitted from the $q^{2}$ distribution in $B \rightarrow \pi l \nu_{l}$ by BaBar Collaboration 18], and the new Belle measurement 13 $B R\left(B \rightarrow \tau \nu_{\tau}\right)=\left(1.06_{-0.28-0.16}^{+0.34+0.18}\right) \times 10^{-4}$ with the interval of QCD predictions $f_{B}=210 \pm 20 \mathrm{MeV}$, it is possible to calculate the $B \rightarrow \pi$ form factor at zero momentum transfer, independent of $\left|V_{u b}\right|$. One obtains:

$f_{B \pi}^{+}(0)=0.24 \pm 0.01 \pm 0.01 \pm 0.04 \pm 0.02$,

where the first three errors are experimental (semileptonic width, shape, leptonic width) and the fourth error is due to the theoretical uncertainty of $f_{B}$. The central value of this estimate nicely agrees with the LCSR prediction but the errors are again too large to exclude a smaller form factor.

\subsection{Inverse moment of $B$-meson DA}

Recently, a new type of LCSR was derived from the correlation function between the vacuum and $\mathrm{B}$ meson, relating the $B \rightarrow \pi$ form factor at small momentum transfer to the $B$-meson DA's 19. A similar approach was suggested in the framework of SCET in 20. In the leading order, this sum rule establishes a simple relation between the combination $\left(m_{B} \lambda_{B} f_{B \pi}^{+}(0) / f_{B}\right)$ and the parameters in the pion channel. (Note that in the above combination the heavy-quark mass dependence drops out in the $m_{b} \rightarrow \infty$ limit). Us- 
ing the results for $f_{B \pi}^{+}(0)$ and $f_{B}$ from the sum rules described in the subsections 3.1 and 3.2, respectively, a new estimate for the inverse moment $\lambda_{B}=460 \pm 160 \mathrm{MeV}$ was obtained, in agreement with the HQET sum rule estimates 21] and with the interval for $\lambda_{B}$ in (2). Note that the product of the form factor and inverse moment is fixed by the new sum rule. The latter is therefore violated if both $f_{B \pi}^{+}(0)$ and $\lambda_{B}$ decrease e.g., up to their lower limits in (2). A more detailed numerical analysis of this relation including the power suppressed corrections is currently in progress.

\section{4. $S U(3)_{f l}$ violation in $B$ decays}

QCD sum rules allow to calculate the differences between the hadronic matrix elements with kaons and pions in terms of $m_{s} \neq m_{u, d}$ and the ratios of the strange- and nonstrange quark condensates. In this way, the ratio $f_{K} / f_{\pi}$ is reproduced and the $S U(3)_{f l}$-asymmetries in the kaon DA's vs pion DA's are predicted (see, e.g. 222 and the recent comprehensive analysis in 23). After including the calculated $S U(3)_{f l}$-violation effects into LCSR, one predicts the ratios of the form factors with and without strange hadrons. The results obtained in 24 have been updated in 22] (after an important change of the first Gegenbauer moment in the twist-2 kaon DA) to the following intervals:

$\frac{f_{B K}^{+}(0)}{f_{B \pi}^{+}(0)}=1.36_{-0.09}^{+0.12}, \quad \frac{f_{B_{s} K}^{+}(0)}{f_{B \pi}^{+}(0)}=1.21_{-0.11}^{+0.14}$.

The $S U(3)_{f l}$-violation effects are large, and they influence the flavour-symmetry relations for nonleptonic charmless $B$-decays. The prediction for $B R\left(B_{s} \rightarrow K^{+} K^{-}\right)$obtained in 2] on the basis of these relations, including the above sum rule predictions for the form factor ratios, have recently been confirmed by CDF Collaboration 25.

\subsection{Hadronic matrix elements for $B \rightarrow \pi \pi$}

An extension of LCSR technique 26 allows to calculate various hadronic matrix elements $\left\langle h_{1} h_{2}\left|O_{i}\right| B\right\rangle^{T}$ and compare them with each other. An important study case is $\bar{B}^{0} \rightarrow \pi^{+} \pi^{-}$. One starts with the correlation function:

$$
F_{\alpha}^{\left(O_{i}\right)}(p, q, k)=-\int d^{4} x e^{-i(p-q) x} \int d^{4} y e^{i(p-k) y}
$$

$$
\times\left\langle 0\left|T\left\{j_{\alpha 5}^{(\pi)}(y) O_{i}(0) j_{5}^{(B)}(x)\right\}\right| \pi^{-}(q)\right\rangle,
$$

where $j_{\alpha 5}^{(\pi)}=\bar{u} \gamma_{\alpha} \gamma_{5} d$ and $j_{5}^{(B)}=m_{b} \bar{b} i \gamma_{5} d$ are the quark currents interpolating the pion and the $B$ meson, respectively. The correlation function $F^{\left(O_{i}\right)}$ is calculated in QCD at large spacelike external momenta squared $(p-k)^{2},(p-q)^{2}, P^{2}=$ $(p-q-k)^{2}$, in a form of OPE with the pion DA's, that is, using the same long-distance input, as in the LCSR for the $B \rightarrow \pi$ form factor (see sect. 3.2). Furthermore, both hard- and softgluon effects are included, contributing to different terms of the OPE. The result for the correlation function is then matched to the hadronic dispersion relations, subsequently in the pion $\left(j_{\alpha 5}^{(\pi)}\right)$ and $B$ meson $\left(j_{5}^{(B)}\right)$ channels. The procedure is formulated in such a way that the final sum rule relation contains the desired matrix element $\left\langle\pi^{+} \pi^{-}\left|O_{i}\right| \bar{B}^{0}\right\rangle$. The calculation is fulfilled at finite $m_{b}$. A further expansion of the sum rule in $1 / m_{b}$ allows to compare the finite $m_{b}$ results for the hadronic matrix elements with the corresponding QCDF predictions, and, moreover, to estimate the uncalculable $1 / m_{b}$ corrections. For a given operator $O_{i}$ in the correlation function (77) various contractions of quark fields are possible. Collecting the lowest contributions to OPE, one identifies the diagrams with emission, penguin and annihilation topologies. If one retains only diagrams with a topology $T$ in the OPE, the sum rule result is interpreted as $\left\langle\pi^{+} \pi^{-}\left|O_{i}\right| \bar{B}^{0}\right\rangle_{T}$. Note that an important step in obtaining the LCSR results for $B \rightarrow \pi \pi$ hadronic matrix elements is the analytical continuation from a large spacelike $P^{2}<0\left(\left|P^{2}\right| \gg \Lambda_{Q C D}^{2}\right)$ to the large timelike $P^{2}=m_{B}^{2}$. The imaginary part (discontinuity) in $P^{2}$ generated by this continuation is identified with the strong phase of the hadronic matrix element in the (local) quark-hadron duality approximation. This duality assumption introduces an additional "systematic" uncertainty.

The details of the procedure and calculation for various topologies can be found in [262715]. In particular, a finite result for the hadronic matrix element $\left\langle\pi^{+} \pi^{-}\left|O_{1}^{u}\right| \bar{B}^{0}\right\rangle_{A}$ of the current-current operator $O_{1}^{u}=\left(\bar{d} \Gamma_{\mu} u\right)\left(\bar{u} \Gamma^{\mu} b\right)$ is obtained, with an imaginary part which contributes to the strong 
phase. The origin of this annihilation phase at the diagram level is explained in [15. In addition, an important factorizable annihilation contribution from the quark-penguin operator $O_{6}$ is found. For the contributions with hard gluons the method suggested in [26] was modified in [15], to avoid complicated two-loop multi-scale diagrams. Instead of performing the QCD calculation of the vacuum-to-pion correlation function (7), one starts from the pion-pion correlator, thereby reducing the calculation to one-loop diagrams. The input parameters used in the LCSR for $B \rightarrow \pi \pi$ hadronic matrix elements are the same as in the LCSR for $f_{B \pi}^{+}$. This form factor determines the factorizable $B \rightarrow \pi \pi$ amplitude, that is, the hadronic matrix element of the $O_{1}^{u}$ in the emission topology:

$\left\langle\pi^{+} \pi^{-}\left|O_{1}^{u}\right| \bar{B}^{0}\right\rangle_{E} \simeq i f_{\pi} f_{B \pi}^{+}(0) m_{B}^{2}$.

The LCSR obtained in 262715] were used to estimate the hadronic matrix elements $\left\langle\pi^{+} \pi^{-}\left|O_{i}\right| \bar{B}^{0}\right\rangle_{T}$ of all effective operators $O_{i}$ (except the electroweak penguin operators) with nonfactorizable emission, penguin and annihilation topologies.

The ratio of the annihilation matrix element to the factorizable amplitude (8), defined as

$r_{A}^{(\pi \pi)}=\frac{\left\langle\pi^{+} \pi^{-}\left|O_{1}^{u}\right| \bar{B}^{0}\right\rangle_{A}}{2\left\langle\pi^{+} \pi^{-}\left|O_{1}^{u}\right| \bar{B}^{0}\right\rangle_{E}}$,

as well as the analogous ratios for other topologies and operators parameterize the nonfactorizable effects. The numerical results are :

* nonfactorizable emission

$$
\begin{array}{r}
r_{E}^{(\pi \pi)}=\left[\left(1.8_{-0.7}^{+0.5}\right) \times 10^{-2}\right]_{\mathrm{soft}} \\
+\left[\left(-1.9_{-0.1}^{+0.5}+i\left(-3.6_{-0.4}^{+1.0}\right)\right) \times 10^{-2}\right]_{\mathrm{hard}},
\end{array}
$$

where the contribution of the hard gluon is a preliminary result (with twist 2 accuracy),

* charming penguin:

$r_{P_{c}}^{(\pi \pi)}=\left[-0.18_{-0.68}^{+0.06}+i\left(-0.80_{-0.08}^{+0.17}\right)\right] \times 10^{-2}$,

* annihilation:

$r_{A}^{(\pi \pi)}=\left[-0.67_{-0.87}^{+0.47}+i\left(3.6_{-1.1}^{+0.5}\right)\right] \times 10^{-3}$.
These results are encouraging for the method because all nonfactorizable effects including their imaginary parts (contributions to the strong phase) are found small, so that the light-cone OPE can be trusted. Moreover, expanding LCSR in $1 / m_{b}$, one reproduces the hierarchy of $\mathrm{QCDF}$, with a possibility to estimate also the $1 / m_{b}$ effects (e.g. the nonfactorizable soft-gluon contributions in the emission topology [26]).

At the same time, the results are rather discouraging for the phenomenology, because sum rules do not reveal any "hidden" large effect in penguin and/or annihilation topologies, thereby supporting the default version of QCDF. Adding up all small effects estimated with LCSR one obtains (for the CKM angle $\left.\gamma=(58.5 \pm 10)^{\circ}\right)$ ):

$$
\begin{aligned}
B R\left(B^{+} \rightarrow \pi^{+} \pi^{0}\right) & =\left(6.6_{-1.3-0.8}^{+1.8+0.8}\right) \times 10^{-6} \\
B R\left(B^{0} \rightarrow \pi^{+} \pi^{-}\right) & =\left(9.7_{-1.9-1.2}^{+2.3+1.2}\right) \times 10^{-6} \\
B R\left(B^{0} \rightarrow \pi^{0} \pi^{0}\right) & =\left(0.25_{-0.08-0.06}^{+0.12+0.07}\right) \times 10^{-6} .
\end{aligned}
$$

The last two predictions significantly differ from the current experimental averages [1] for $B R\left(B^{0} \rightarrow \pi^{+} \pi^{-}\right)$and $B R\left(B^{0} \rightarrow \pi^{0} \pi^{0}\right)$. For brevity I do not show the LCSR results for $C P$ asymmetries presented in [15, where a very small direct asymmetry for $B^{0} \rightarrow \pi^{+} \pi^{-}$is predicted.

The comparison with the data based on the most general isospin expansion of the decay amplitudes [15] reveals that the isospin-two amplitude determining the $B^{-} \rightarrow \pi^{-} \pi^{0}$ decay is in a reasonable agreement with theoretical predictions, (using the $B \rightarrow \pi$ form factor calculated from LCSR), whereas one needs additional contributions to the isospin-zero amplitude generated by the $\Delta I=1 / 2$ pieces of the effective Hamiltonian.

\section{CONCLUSIONS}

QCD sum rules for $f_{B}$ and LCSR for $B \rightarrow \pi$ form factor agree with the lattice QCD results, and hint at a smaller value of $\left|V_{u b}\right|$ than the "inclusive" one, but the uncertainties remain too large for a decisive comparison. A scenario with both small $f_{B \pi}^{+}(0)$ and $\lambda_{B}$ is disfavored by the new sum rule for the product of these two parameters. LCSR calculations of hadronic 
matrix elements $\left\langle\pi \pi\left|O_{i}\right| B\right\rangle_{T}$ reveal suppressed nonfactorizable effects with small strong phases in $B \rightarrow \pi \pi$ (probably also in $B \rightarrow K \pi$, a more detailed analysis including $S U(3)_{f l}$-violation effects is in progress). Thus, the origin of an additional isospin-zero amplitude and related large strong phase indicated by the $B \rightarrow \pi \pi$ data $^{2}$ cannot be identified.

I thank Giulia Ricciardi and other organizers for a very interesting and enjoyable workshop. I gratefully acknowledge collaboration with Thomas Mannel, Blazenka Melić, Martin Melcher and Nils Offen on the topics discussed in this talk. This work is supported by Deutsche Forschungsgemeinschaft (DFG) under the project DFG KH205/1-1.

\section{REFERENCES}

1. [Heavy Flavor Averaging Group (HFAG)], arXiv:hep-ex/0603003

2. A. J. Buras, R. Fleischer, S. Recksiegel and F. Schwab, Nucl. Phys. B 697 (2004) 133.

3. M. Beneke, G. Buchalla, M. Neubert and C. T. Sachrajda, Phys. Rev. Lett. 83 (1999) 1914; Nucl. Phys. B 606 (2001) 245.

4. C. W. Bauer, D. Pirjol, I. Z. Rothstein and I. W. Stewart, Phys. Rev. D 70 (2004) 054015; C. W. Bauer, I. Z. Rothstein and I. W. Stewart, arXiv:hep-ph/0510241 J. Chay and C. Kim, Nucl. Phys. B 680 (2004) 302.

5. M. Beneke and S. Jäger, arXiv:hep-ph/0512351

6. Y. Y. Keum and H. n. Li, Phys. Rev. D 63 (2001) 074006; Y. Y. Keum, H. n. Li and A. I. Sanda, Phys. Lett. B 504 (2001) 6; H. n. Li and S. Mishima, Phys. Rev. D 73 (2006) 114014.

7. M. Ciuchini, E. Franco, G. Martinelli and L. Silvestrini, Nucl. Phys. B 501, 271 (1997); M. Ciuchini, E. Franco, G. Martinelli, A. Masiero, M. Pierini and L. Silvestrini, arXiv:hep-ph/0407073

8. C. M. Arnesen, Z. Ligeti, I. Z. Rothstein and I. W. Stewart, arXiv:hep-ph/0607001
9. T. Feldmann and T. Hurth, JHEP 0411 (2004) 037.

10. M. Jamin and B. O. Lange, Phys. Rev. D 65 (2002) 056005.

11. A. A. Penin and M. Steinhauser, Phys. Rev. D 65 (2002) 054006.

12. M. Okamoto, PoS LAT2005 (2006) 013 arXiv:hep-lat/0510113.

13. K. Ikado et al. [Belle Collaboration] arXiv:hep-ex/0604018

14. P. Ball and R. Zwicky, Phys. Rev. D 71 (2005) 014015 ; hep-ph/0507076.

15. A. Khodjamirian, T. Mannel, M. Melcher and B. Melić, Phys. Rev. D 72 (2005) 094012.

16. M. C. Arnesen, B. Grinstein, I. Z. Rothstein and I. W. Stewart, Phys. Rev. Lett. 95 (2005) 071802.

17. T. Becher and R. J. Hill, Phys. Lett. B 633, 61 (2006).

18. B. Aubert et al. [BABAR Collaboration], Phys. Rev. D 72 (2005) 051102

19. A. Khodjamirian, T. Mannel and N. Offen, Phys. Lett. B 620 (2005) 52.

20. F. De Fazio, T. Feldmann and T. Hurth, Nucl. Phys. B 733 (2006) 1.

21. A. G. Grozin and M. Neubert, Phys. Rev. D 55 (1997) 272; V. M. Braun, D. Y. Ivanov and G. P. Korchemsky, Phys. Rev. D 69, 034014 (2004).

22. A. Khodjamirian, T. Mannel and M. Melcher, Phys. Rev. D 70, 094002 (2004)

23. P. Ball, V. M. Braun and A. Lenz, JHEP 0605, 004 (2006) arXiv:hep-ph/0603063.

24. A. Khodjamirian, T. Mannel and M. Melcher, Phys. Rev. D 68 (2003) 114007.

25. A. Abulencia [CDF Collaboration], arXiv:hep-ex/0607021

26. A. Khodjamirian, Nucl. Phys. B 605 (2001) 558 .

27. A. Khodjamirian, T. Mannel and P. Urban, Phys. Rev. D 67 (2003) 054027; A. Khodjamirian, T. Mannel and B. Melić, Phys. Lett. B 571 (2003) 75 .

28. A. B. Kaidalov and M. I. Vysotsky, arXiv:hep-ph/0603013

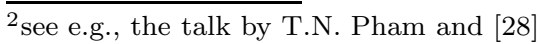

